\title{
Bone pain palliation outcomes and possibility of Radium-223 re-treatment in mCRPC
}

\author{
Viviana Frantellizzi ${ }^{1}$, Julia Lazri ${ }^{2}$, Mariano Pontico ${ }^{3}$, Arianna Pani ${ }^{4}$, Giuseppe De Vincentis ${ }^{2}$ \\ ${ }^{1}$ Department of Molecular Medicine, Sapienza University of Rome, Rome, Italy; \\ ${ }^{2}$ Department of Radiological Sciences, Oncology and Anatomical Pathology, Sapienza University of Rome, Rome, Italy; \\ ${ }^{3}$ Ph.D., Program in Morphogenesis \& Tissue Engineering, "Sapienza" University of Rome, Rome, Italy; \\ ${ }^{4}$ Clinical Pharmacology and Toxicology, University of Milan, Milan, Italy.
}

\begin{abstract}
Summary $\quad$ Objective. Bone secondary localizations from metastatic castration-resistant prostate cancer are associated with an increase in mortality and $a$ reduction in the patient's quality of life. Radium-223 is a targeted alpha-therapy approved for the treatment of $m C R P C$ (metastatic castration resistant prostate cancer) patients with symptomatic bone metastases. To our knowledge, no previous study has been performed assessing the bone pain palliation outcomes following Radium-223 therapy.

Materials and Methods. A mCRPC patient with symptomatic bone localizations and relevant bone pain symptoms has been subjected to Radium-223 treatment. Pain was assessed over time from the first administration of Radium-223 to follow-up. Results. After Radium-223 treatment, patient showed a signifcant BPI (Brief Pain Inventory) decline from 7 to 4 and a concomitant partial regression of multiple bone hot spots in the bone scan exam. Three months after the last infusion of Radium-223, further BPI decline (from 4 to 2) with bone scan depicting stable disease was observed. However, after 6 months from Radium-223 treatment end, BPI increased from 2 to 10. Conclusions. Taking into account the effectiveness on bone pain relief and the low toxicity profile showed by Radium-223 treatment, we encourage further analysis on large cohort to investigate the clinical outcome after Radium-223 treatment, in terms of bone pain palliation, together with the possibility of Radium-223 re-treatment in selected patients..
\end{abstract}

KEY WORDS: Radium-223 dichloride; Bone pain; Palliative treatment; $m C R P C$.

Submitted 28 January 2020; Accepted 18 February 2020

\section{INTRODUCTION}

Prostate cancer (PCa) in men worldwide is the second most common cancer $(1,2)$. Most subjects with metastatic PCa, following a median of 18-24 months of endocrine therapy, no longer respond to traditional androgen deprivation therapy $(\mathrm{ADT})(3,4)$ and are categorized as castration-resistant prostate cancer (CRPC), leading to disease progression $(5,6)$. CRPC disease progression is characterized by the presence of pain-inducing bone metastases ( $90 \%$ of patients), with the increase in total alkaline phosphatase (tALP) and prostate-specific antigen (PSA) levels $(7,8)$. The development of bone metastases is associated with an increase in mortality and a reduction in the patient's health-related quality of life (QoL) (9-11). Treatment options for metastatic CRPC
(mCRPC) have recently and include cytotoxic therapy (e.g., docetaxel, cabazitaxel), immunotherapy, oral hormonal therapies targeting the androgen receptor axis (e.g., enzalutamide and abiraterone), bone health agents (e.g., denosumab and zoledronic acid) and targeted alpha-therapy (Radium-223 dichloride) (12-14). Radium223 has been approved for the treatment of patients with mCRPC with symptomatic bone metastases and no known visceral metastatic disease (15). This radiopharmaceutical have a dual mechanism of action, destroying bone-metastatic cancer cells and affecting tumor-induced pathological bone activity (16). Currently, Radium-223 is approved as monotherapy or in combination with a Luteinizing Hormone-Releasing Hormone (LHRH) analogue for the treatment of adult patients with MCRPC who are in progression after at least two prior lines of systemic therapy (other than LHRH analogues), or ineligible for any available systemic mCRPC treatment (17). Pharmacovigilance Risk Assessment Committee (PRAC) of European Medicines Agency (EMA) carried out a review on Radium-223 after data from a clinical study suggested that patients treated with Radium-223 in combination with abiraterone and prednisone/prednisolone could be at risk of dying earlier and had more fractures than patients given placebo with abiraterone and prednisone/prednisolone (18). The above recommendations are based on the assessment of data resulting from a randomized, double-blind, placebo-controlled phase III trial (ERA-223), which showed an increased incidence of skeletal-related events (SRE) (28.6\% vs $11.4 \%$ ), a possible reduction in median overall survival (OS) (30.7 months vs 33.3 months, HR 1.195, p = 0.13) and an increased risk of radiological non-bone progression ( HR 1.376, $\mathrm{p}=0.07$ ) among patients receiving Radium-223 in combination with abiraterone acetate plus prednisone/prednisolone (n $=401$ ) compared to patients receiving placebo in combination with abiraterone acetate plus prednisone/prednisolone $(n=405)$ (19). Despite several clinical trials and prospective studies have been carried out aiming to investigate the outcomes of Radium-223 therapy in terms of OS, progression-free survival (PFS), SRE and multiple QoL parameters (20), to our knowledge, in the literature there are no studies assessing specifically the bone pain palliation outcomes, as an independent factor, following the end of Radium-223 treatment. The following study 
describes a mCRPC patient, presenting a significant reduction of bone pain during the therapy, followed by further bone pain palliation after the first 3 months following the end of Radium-223 therapy without modification of pain-relieving medications.

\section{MATERIALS AND METHOdS}

A 77 years-old man was diagnosed with PCa (Gleason score 9, 4+5) in February 2014. He began ADT with Triptorelin and Bicalutamide for 19 months. Furthermore, after 2 years from the diagnosis, he has been treated with external beam radiation therapy (EBRT) to secondary bone lesions on the right pelvis. He came to our attention in November 2016 with a PSA recurrence (PSA $168 \mathrm{ng} / \mathrm{mL}$ ) despite ADT, his testosterone level was $0.34 \mathrm{ng} / \mathrm{dL}$ ), therefore he was diagnosed with mCRPC. The patient presented positive 99mTc-HDP bone scan imaging with increased uptake in the cranium, in both scapulae, in the left humerus and radius, in multiple ribs and vertebrae, in the pelvis and in the left femur (Figure 1A). The CT scan was negative for visceral metastasis. He was defined as a poly-metastatic patient (6-20 metastatic foci). Therefore, we started Radium-223 treatment. The Brief Pain Inventory by Numeric Rating Scale (BPI) value was 7 in spite of nonsteroidal antiinflammatory drug (NSAID) (ketorolac) and acetaminophen treatment.

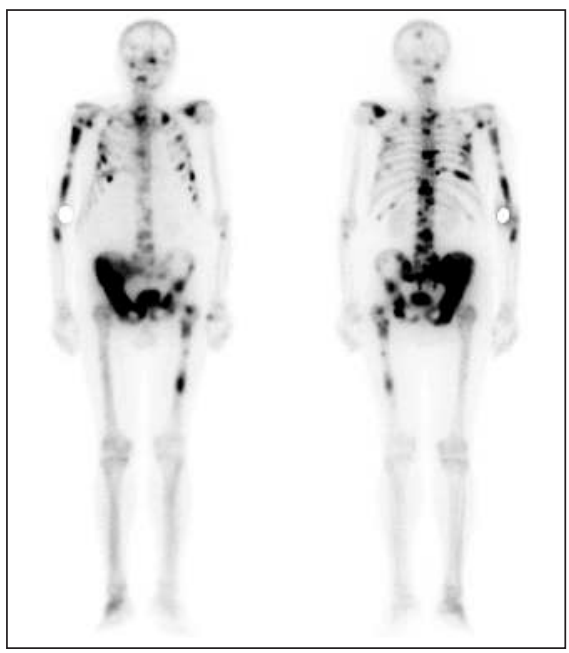

Figure 1A. Baseline 99mTc-HDP bone scan imaging. showed progression in the $99 \mathrm{mTc}$-HDP bone scan performed in October 2017, after 6 months from the end of the Radium-223 therapy (Figure 1C), with extension of the areas of pathological uptake previously reported. Unfortunately, after 13 months of follow-up, the patient died for multi-organ failure.

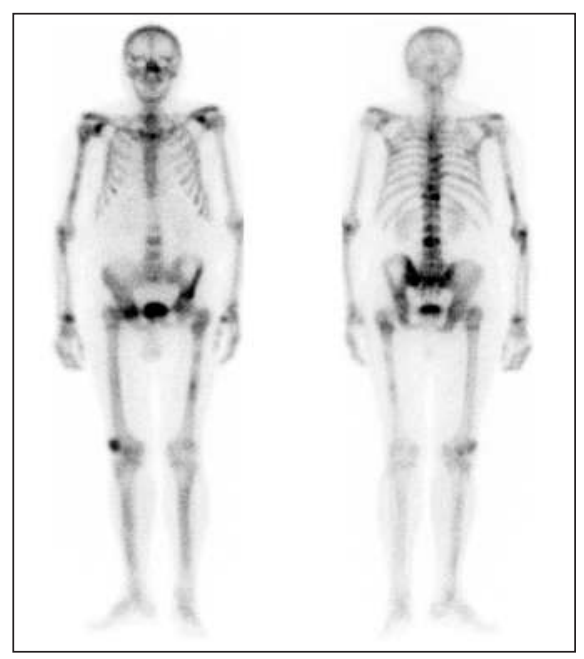

Figure 1B.

End-of treatment $99 m T c-H D P$ bone scan imaging.

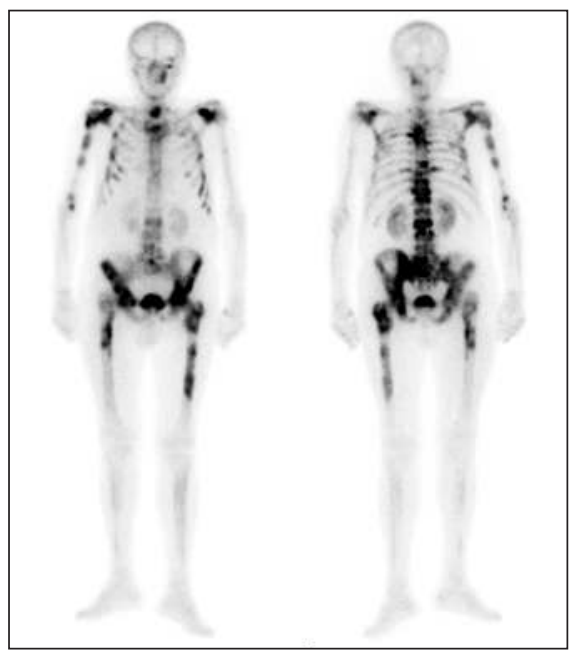

Figure 1C.

99mTc-HDP bone scan imaging at 6 months follow-up.

\section{Results}

After 6 cycles of Radium-223 treatment, BPI declined from 7 to 4 without modification of pain-relieving medications. The bone scan performed in April 2017 showed a partial regression of multiple bone hot spots (Figure 1B).

The patient reported further improvement with BPI that declined from 4 to 2 after 3 months from the last administration of Radium-223 treatment with stable disease resulting in the bone scan. Nevertheless, at 6 months follow-up BPI increased from 2 to 10 without modification of pain-relieving medications. Pain score measurements during and after the Radium-223 treatment are shown in Figure 2. The patient
Figure 2.

BPI trend over time.

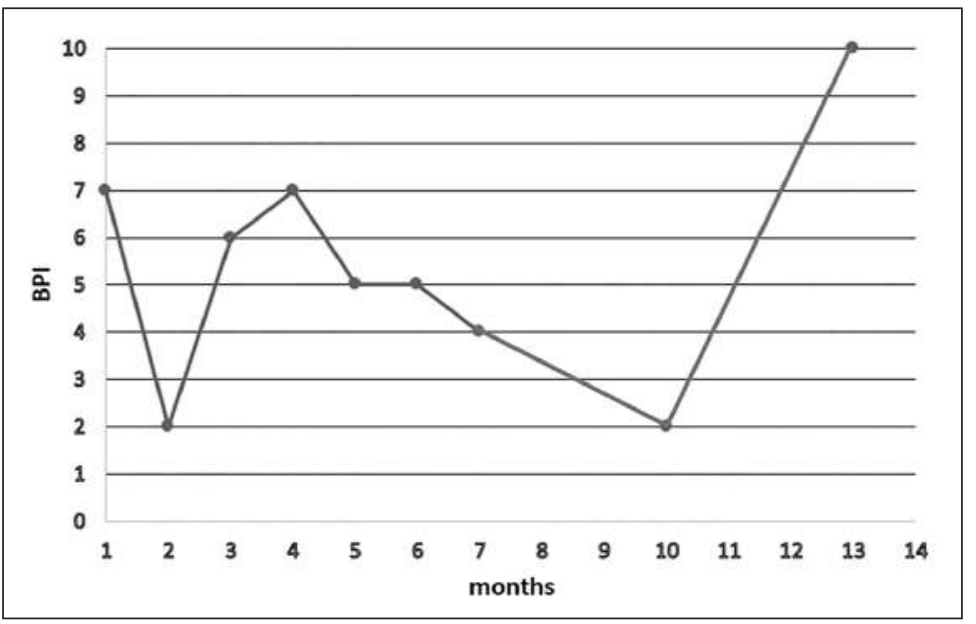




\section{Discussion}

In this study, we presented the case of an MCRPC patient treated with Radium-223, showing bone pain reduction during the therapy which continued with a further decline for the next 3 months after the end treatment. Nevertheless, after 6 months from Radium-223 treatment, BPI suddenly increased from 2 to 10 with the radiological progression of disease described in several bone districts, revealed with bone scan.

The phase 3 trial ALSYMPCA showed a significant effect of Radium-223 on OS and a delay in median time to the first SRE (21). In the meantime, several retrospective studies reported a difference in median OS between patients who received 1 to 4 versus 5 to 6 injections, demonstrating that a higher number of Radium-223 injections was associated with prolonged OS (median OS of 6.2 months, versus 17.9 months) (22). ALSYMPCA show furthermore that Radium-223 patients reduced the risk of need for EBRT for bone pain and notably delayed the time to first use of opioids (23). This is associated with significant palliation of bone pain by the radiopharmaceutical. However, ALSYMPCA was not planned to evaluate the effect of Radium-223 on pain; any response to pain observed or lack of it was not considered a reason to stop treatment with Radium-223. The effect of treatment on pain was not systematically documented as it was not one of the objectives of the study. Currently, patients receive up to six intravenous injections of Radium-223, $55 \mathrm{kBq} / \mathrm{kg}$ in 4-week intervals. There have been recent studies of retreatment with Radium-223 (24). One of these is the open-label, phase 1/2 study (NCT01934790) (25).

This study, including 44 patients with no disease progression in bone during the first treatment and presence of later progression, that received Radium-223 re-treatment, up to 6 additional Radium-223 injections, showed that retreatment with Radium-223 was well tolerated with favorable effects on disease progression. Another ongoing phase II study (NCT02023697) evaluated standard dose (55 kBq/kg every 4 weeks up to 6 injections) versus high dose ( $88 \mathrm{kBq} / \mathrm{kg}$ every 4 weeks up to 6 injections) and versus extended standard dose $(55 \mathrm{kBq} / \mathrm{kg}$ every 4 weeks up to 12 injections) $(26,27)$. In literature has been already showed how Radium-223 could significantly extend OS, associated with a delay in median time to the first symptomatic SRE, meaningful improvement in QoL and bone pain palliation $(23,28)$. Several trials showed that Radium-223 is well tolerated and has a favorable hematologic safety profile (29), with a low incidence of myelosuppression (30). Such safety is confirmed from long-term safety ALSYMPCA analysis up to 3 years from first injection, which indicated that Radium-223 remained still well tolerated, with low myelosuppression incidence and no new safety concerns (31). It is possible to postulate that some patients after initial treatment may derive benefit from extended treatment with Radium-223.

The open-label, phase 1/2 NCT01934790 trial (25) showed that re-treatment with Radium-223 sustained benefit on disease and progression was well tolerated. In particular, it showed a low incidence of clinical events such as symptomatic SRE or radiographic bone progression. Furthermore, in this study tALP declined from baseline values after re-treatment, suggesting a continuation of the biologic effects of Radium-223; moreover, the median OS increased to 24.4 months at the end of the 2-year active follow-up period. A further study focused on these patients confirms its safety in a 2-year active follow-up analysis, especially with regards to the minimal hematologic toxicity and efficacy outcome from 2-year follow-up of the retreatment with Radium-223. In view of these remarkable results and based on our wide experience, we could reasonably assume that this patient would have provided clinical benefits if he continued the Radium-223 therapy.

\section{Conclusions}

Further analysis should be conducted on a large cohort to evaluate the clinical outcome in terms of bone pain palliation after Radium-223 treatment and the possibility, considering the low toxicity profile, of re-treatment in selected patients.

\section{Ethical APPROVAL}

This study has been approved by the local ethical committee.

All procedures performed in studies involving human participants were in accordance with the ethical standards of the institutional and/or national research committee and with the 1964 Helsinki declaration and its later amendments or comparable ethical standards.

\section{INFORMED CONSENT}

Informed consent was obtained from all individual participants included in the study.

\section{RESEARCH INVOLVING HUMAN PARTICIPANTS AND/OR ANIMALS}

This article does not contain any studies with animals performed by any of the authors.

\section{REFERENCES}

1. Siegel RL, Miller KD, Jemal A. Cancer statistics, 2016. CA Cancer J Clin. 2016; 66:7-30

2. Santer FR, Erb HH, McNeill RV. Therapy escape mechanisms in the malignant prostate. Semin Cancer Biol. 2015; 35:133-44.

3. Sciarra A, Gentilucci A, Silvestri I, et al. Androgen receptor variant 7 (AR-V7) in sequencing therapeutic agents for castratrion resistant prostate cancer: A critical review. Medicine (Baltimore) 2019; 98:e15608

4. Ricci M, Frantellizzi V, Bulzonetti N, De Vincentis G. Reversibility of castration resistance status after Radium-223 dichloride treatment: clinical evidence and Review of the literature. Int J Radiat Biol. 2019; 95:554-561.

5. Cornford P, Bellmunt J, Bolla M, et al. EAU-ESTRO-SIOG Guidelines on prostate cancer. Part II: treatment of relapsing, metastatic, and castration-resistant prostate cancer. Eur Urol. 2017; 71:630-42.

6. Lowrance WT, Roth BJ, Kirkby E, et al. Castration-resistant prostate cancer: AUA guideline amendment 2015. J Urol. 2016; 195:1444-52. 
7. Sathiakumar N, Delzell E, Morrisey MA, et al. Mortality following bone metastasis and skeletal-related events among men with prostate cancer: a population-based analysis of US Medicare beneficiaries, 1999-2006. Prostate Cancer Prostatic Dis. 2011; 14:177-83.

8. Som A, Tu SM, Liu, et al. Response in bone turnover markers during therapy predicts overall survival in patients with metastatic prostate cancer: analysis of three clinical trials. Br J Cancer. 2012; 107:1547-53.

9. Smith HS. Painful osseous metastases. Pain Physician 2011; 14:E373-403.

10. De Vincentis G, Frantellizzi V, Follacchio GA, et al. No evidence of association between psychological distress and pain relief in patients with bone metastases from castration-resistant prostate cancer treated with 223Radium. Eur J Cancer Care (Engl) 2019; 28:e13112.

11. De Vincentis G, Monari F, Baldari S, et al. Narrative medicine in metastatic prostate cancer reveals ways to improve patient awareness \& quality of care. Future oncology (London, England) 2018; 14:2821-32.

12. Asselah J, Sperlich C. Post-docetaxel options for further survival benefit in metastatic castration-resistant prostate cancer: Questions of choice. Can Urol Assoc J. 2013; 7:S11-7.

13. Heidenreich A, Bastian PJ, Bellmunt J, et al. EAU guidelines on prostate cancer. Part II: Treatment of advanced, relapsing, and castration-resistant prostate cancer. Eur Urol. 2014; 65:467-79.

14. De Vincentis G, Gerritsen W, Gschwend JE, et al. Advances in targeted alpha therapy for prostate cancer. Ann Oncol. 2019; 30:1728-1739.

15. Baldari S, Boni G, Bortolus R, et al. Management of metastatic castration-resistant prostate cancer: A focus on radium-223: Opinions and suggestions from an expert multidisciplinary panel. Crit Rev Oncol Hematol. 2017; 113:43-51.

16. Henriksen G, Breistol K, Bruland OS, et al. Significant antitumor effect from bone-seeking, alpha-particle-emitting (223)Ra demonstrated in an experimental skeletal metastases model. Cancer Res. 2002; 62:3120-5.

17. Du Y, Carrio I, De Vincentis $G$, et al. Practical recommendations for radium-223 treatment of metastatic castration-resistant prostate cancer. Eur J Nucl Med Mol Imaging. 2017; 44:1671-8.

18. O'Sullivan JM, Heinrich D, James ND, et al. The case against the European Medicines Agency's change to the label for Radium223 for the treatment of metastatic castration-resistant prostate cancer. Eur Urol. 2019; 75:e51-e2.

19. Smith M, Parker C, Saad F, et al. Addition of radium-223 to abiraterone acetate and prednisone or prednisolone in patients with castration-resistant prostate cancer and bone metastases (ERA 223): a randomised, double-blind, placebo-controlled, phase 3 trial. The Lancet. Oncology. 2019; 20:408-19.

20. Saad F, Gillessen S, Heinrich D, et al. Disease characteristics and completion of treatment in patients with metastatic castrationresistant prostate cancer treated with Radium-223 in an international early access program. Clin Genitourin Cancer. 2019; 17:348$355 . e 5$

21. Parker C, Nilsson S, Heinrich D, et al. Alpha emitter Radium223 and survival in metastatic prostate cancer. N Engl J Med. 2013; $369: 213-23$

22. Sartor O, Coleman R, Nilsson S, et al. Effect of radium-223 dichloride on symptomatic skeletal events in patients with castration-resistant prostate cancer and bone metastases: results from a phase 3, double-blind, randomised trial. The Lancet. Oncology. $2014 ; 15: 738-46$

23. Nilsson S, Cislo P, Sartor O, et al. Patient-reported quality-oflife analysis of radium-223 dichloride from the phase III ALSYMPCA study. Ann Oncol. 2016; 27:868-74.

24. Sartor AO, Heinrich D, Mariados N, et al. Radium-223 (Ra223) re-treatment (Re-tx): First experience from an international, multicenter, prospective study in patients (Pts) with castration-resistant prostate cancer and bone metastases (mCRPC). Journal of Clinical Oncology. 2016; 34(Suppl 2):197.

25. Sartor O, Heinrich D, Mariados N, et al. Re-treatment with radium-223: 2-year follow-up from an international, open-label, phase $1 / 2$ study in patients with castration-resistant prostate cancer and bone metastases. Prostate. 2019; 79:1683-91.

26. Parker CC, Pascoe S, Chodacki A, et al. A randomized, doubleblind, dose-finding, multicenter, phase 2 study of radium chloride (Ra 223) in patients with bone metastases and castration-resistant prostate cancer. Eur Urol. 2013; 63:189-97.

27. NCT02023697. Radium-223 Dichloride $50 \mathrm{kBq} / \mathrm{kg}(55 \mathrm{kBq} / \mathrm{kg}$ After Implementation of NIST Update) Versus $80 \mathrm{kBq} / \mathrm{kg}$ ( $88 \mathrm{kBq} / \mathrm{kg}$ After Implementation of NIST Update), and Versus $50 \mathrm{kBq} / \mathrm{kg}(55$ $\mathrm{kBq} / \mathrm{kg}$ After Implementation of NIST Update) in an Extended Dosing Schedule in Subjects With Castration-resistant Prostate Cancer Metastatic to the Bone. https://clinicaltrials.gov/ct2/show/ results/NCT0202369.

28. Nilsson S, Larsen RH, Fossa SD, et al. First clinical experience with alpha-emitting radium-223 in the treatment of skeletal metastases. Clin Cancer Res. 2005; 11:4451-9.

29. Prelaj A, Rebuzzi SE, Buzzacchino F, et al. Radium-223 in patients with metastatic castration-resistant prostate cancer: efficacy and safety in clinical practice. Oncol Lett. 2019; 17:1467-76.

30. De Vincentis G, Follacchio GA, Frantellizzi V, et al. 223Radichloride therapy in an elderly bone metastatic castration-resistant prostate cancer patient: a case report presentation and comparison with existing literature. Aging Clin Exp Res. 2018; 30:677-680.

31. Vogelzang NJ, Coleman RE, Michalski JM, et al. Hematologic Safety of Radium-223 Dichloride: Baseline Prognostic Factors Associated With Myelosuppression in the ALSYMPCA Trial. Clin Genitourin Cancer. 2017; 15:42-52.e8.

\section{Correspondence}

Viviana Frantellizzi, MD, PhD

Viviana.frantellizzi@uniromal.it

Department of Molecular Medicine, Sapienza University of Rome Viale Regina Elena 324, 00161 Rome (Italy)

Julia Lazri, MD

Julia.lazri@uniromal.it

Giuseppe De Vincentis, MD, PhD

Giuseppe.devincentis@uniromal.it

Department of Radiological Sciences, Oncology and Anatomical Pathology, Sapienza University of Rome, Viale Regina Elena 324, Rome (Italy)

Mariano Pontico, MD

Mariano.pontico@uniromal.it

Ph.D Program in Morphogenesis \& Tissue Engineering,

"Sapienza" University of Rome, Viale Regina Elena 324, Rome (Italy)

Arianna Pani, MD

Arianna.pani@gmail.com

Clinical Pharmacology and Toxicology, University of Milan,

L.go De Benedetti 1, Milan (Italy) 